\title{
A Smart Assistance System for Visually Impaired People
}

\author{
Muppla Jagadeesh, P. Ajay Kumar Reddy, S. Nanda Kishor
}

\begin{abstract}
Resolving the problems of individuals with Visual, Hearing, and Vocal Impairment through a solitary serving framework could be a tough task. Various current investigations focus on the resolution of the problems of 1 of them on top of challenges however not all. The work centers around chase down a noteworthy procedure that guides the externally weakened by permitting them to listen to what's self-addressed as text and it's accomplished by the tactic that catches the image through a camera and converts the content accessible as voice signals. This planned framework provides a path to people with Hearing weakening to image scan that is in morphology by discourse to message modification procedure and that we, in addition, provides a route to the vocally disabled to handle their voice by the guide of text to voice transformation strategy. each one of those 3 arrangements was regulated to be in an exceedingly solitary exceptional framework. each one of those exercises consists of the employment of Raspberry Pi. The outwardly barred individual's unit of measurement is helped by the cycle whereby the image to text and text to discourse is given by the Tesseract OCR (online character acknowledgment). The deaf individuals assist with the cycle of associate application that creates them grasp what the individual says is also shown attributable to the message. Vocally hindered individuals can pass on their message by text. Therefore totally different individuals will hear the message in an exceeding speaker.
\end{abstract}

Keywords: Raspberry Pi, Webcam, Optical Character Recognition, Text to Speech Engine, amplifier.

\section{INTRODUCTION}

A Apparently weakened people report fluctuated difficulties with coming to composed correspondence using existing development, consolidating issues with the course of action, focus, precision, mobility, and adequacy. we have a tendency to gift an awesome comfort that helps the debilitated that effectively and capably scrutinizes the paper printed text. The arranged task uses the technique of a camera-based valuable accommodation which will be utilized by people to see Text records. The construction is for the shutting picture-getting method in Associate in nursing embedded structure-guided into the Raspberry Pi board. The arrangement is moved by groundwork assessments with

Manuscript received on August 27, 2021.

Revised Manuscript received on October 25, 2021.

Manuscript published on October 30, 2021.

* Correspondence Author

Muppla Jagadeesh*, M.Tech (Embedded Systems), Kuppam Engineering College, Kuppam (A.P), India.

Mr. P. Ajay Kumar Reddy, Associate Professor, Department of Electronics and Communication Engineering, Kuppam Engineering College, Kuppam (A.P), India.

Dr. S. Nanda Kishor, Associate Professor, Department of Electronics and Communication Engineering, Kuppam Engineering College, Kuppam (A.P), India.

(C)The Authors. Published by Blue Eyes Intelligence Engineering and Sciences Publication (BEIESP). This is an open access article under the CC BY-NC-ND license (http://creativecommons.org/licenses/by-nc-nd/4.0/) ostensibly banished people, and it is the confined scale and adaptability, that enables stacks of savvy action with the limited game plan. all through this endeavor, we have arranged a substance examined out a system for the trial. The arranged joined system alternatives a camera as Associate in nursing data comfort to require the consideration of the composed correspondence chronicle for digitization and furthermore the separated record is taken care of by an item module the OCR (optical person affirmation engine). away is dead to the affirmation progression of characters and also the street of learning. As an element of the product progression, the Open CV (Open give convenient PC Vision) libraries are familiar with picture catch of text, to attempt to the person affirmation. Partner in Nursing outsized piece of the entranceway development devices worked for people with vision abandons and limited vision are upheld by the two rudimentary construction squares of OCR programming and Text-to-Speech (TTS) engines. Optical person affirmation (OCR) is that the translation of got photographs of composed correspondence into machine-encoded text. OCR is additionally a cycle that associates delegate significance with objects (letters, film numbers) with the picture of a personality. It's portrayed because of the methodology toward dynamic over-checked photographs of machine-printed into a compact PC cooperation fit association. Optical Character affirmation is in like manner supportive for apparently blocked the individuals who can't inspect Text records, in any case, need to get to the substance of the text documents. Optical Character affirmation is utilized to digitalize and imitate messages that are made with the non-motorized structure. Digitizing compositions in like manner diminishes further regions. Disinfection and reproduction a Text record that was engraved on paper is drawn-out and work truly. it's constantly familiar with change over books and files into electronic records to be utilized away and report examination. OCR makes it possible to utilize methods, for instance, machine translation, text to talk, and text mining to the catch/really look at the page. The last seen content record is dealt with to the yield contraptions depending upon the choice of the benefactor. The yield comfort is normally a recipient related with the raspberry pi or a speaker which can legitimize the substance report commotion.

\section{LITERATURE SURVEY}

Beam Kurzweil et.al [1] proposes A K-Reader Mobile assortment of moveable perusing partner's region units planned explicitly for the outwardly disabled. "K-Reader Mobile" might be a portable application that allows the client to filter mail, receipts, flyers, and another record. In any case, these frameworks/gadgets neglect to give partner financial goals to the matter and region unit reachable on explicit stages.

\section{Published By:}

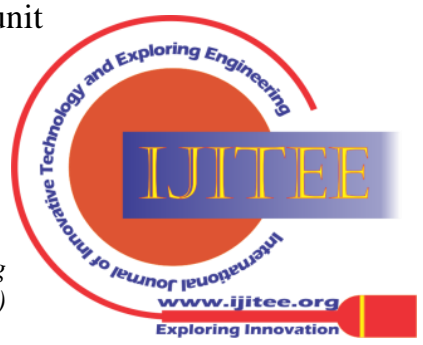


Athira Panicker et.al [2] proposes a reasonable looking partner name perusing framework with voice yield for the undertaking focuses on The archive to be output ought to be almost level, put on a straightforward, dull surface, and content to a great extent dark text composed on a white foundation and it doesn't examine from cutting edge foundations. Marut Tripathi et.al [3] proposes A Navigation System for blind people to explore securely and rapidly, inside the framework deterrent identification and acknowledgment are finished through unhearable sensors and a USB camera. This strategy distinguishes hindrances up to $300 \mathrm{~cm}$ through unhearable sensors and sends input inside the sort of a blaring sound by means of headphones to tell the individual with respect to the deterrent. Dimitrios Dakopoulos et.al [4] proposes A wearable Obstacle evading Electronic Travel Aids for Blind that presents a similar overview among versatile/wearable impediment identification/evasion frameworks (a subcategory of ETAs) in a preliminary to tell the examination local area and clients with respect to the capacities of those frameworks and in regards to the advancement in accommodating innovation for outwardly debilitated people. X.Chen et.al [5] proposes Automatic discovery-related acknowledgment of signs from regular scenes we will in general gift a way to deal with programmed identification and acknowledgment of signs from normal scenes and its application to an indication interpretation task. we have applied the methodology in fostering a Chinese sign interpretation framework, which may precisely notice and recognize Chinese signs as contribution from a camera, and make an interpretation of the perceived text into English in any case, it exclusively works inside the Chinese language. William A. Ainsworth [6] proposes a framework for changing English text into discourse the practicability of changing English text into discourse abuse a sensible pc and a little amount of keep information has been researched anyway it's not fitting for all memory differ of PCs. Zoran Zivkovic et.al [7] proposes partner further developed variation Gaussian Mixture Model for Background Subtraction might be a typical pc vision task. We will in general examine the standard pixel-level methodology. We will in general foster partner affordable variation rule abuse Gaussian combination probability thickness. Algorithmic conditions region unit acclimated ceaselessly update the boundaries and anyway conjointly to simultaneously pick the satisfactory assortment of parts for each image component.

\section{BLOCK DIAGRAM OF PROPOSED METHOD}

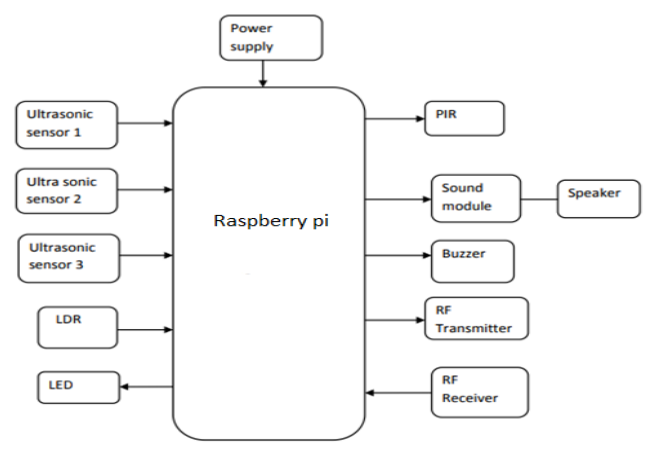

Figure 1: Block diagram of A Smart Assistance System for Visually Impaired people visually impaired abuse of the raspberry pi.- This

The structure of the arranged venture is that the raspberry pi board. The raspberry pi B+ could likewise be one board PC that has four USB ports, an Associate in Nursing PC network port for net alliance, forty GPIO pins for input/yield, CSI camera interface, HDMI port, DSI show interface, SOC (framework on a chip), local space network regulator, Coyote State card opening, sound jack, and RCA video attachment and $5 \mathrm{~V}$ little USB association. the forced offer is given to the $5 \mathrm{~V}$ little USB association of raspberry pi through the Switched Mode Power offer (SMPS). The SMPS changes over the 230V AC gives to 5V DC. net camera is associated with the USB port of raspberry pi. The raspberry pi has an Associate in Nursing OS named RASPION that procedure the changes. The sound yield is taken from the sound jack of the raspberry pi. The renewed discourse yield is enhanced double-dealing Associate in Nursing electronic gear. the online is associated with the PC network port in raspberry pi. The page to peruse is set on a base and conjointly the camera is focused on catching the picture. The caught picture is prepared by the OCR bundle place in raspberry pi. The caught picture is renewed to message by the bundle. The text is renewed into discourse by the TTS motor. the last word yield is given to the electronic hardware from that it's associated with the speaker. Speakers may also get supplanted by Associate in Nursing headphones for accommodation.

\section{HARDWARE AND SOFTWARE REQUIREMENTS HARDWARE REQUIREMENTS}

\section{ARM11 Raspberry Pi board}

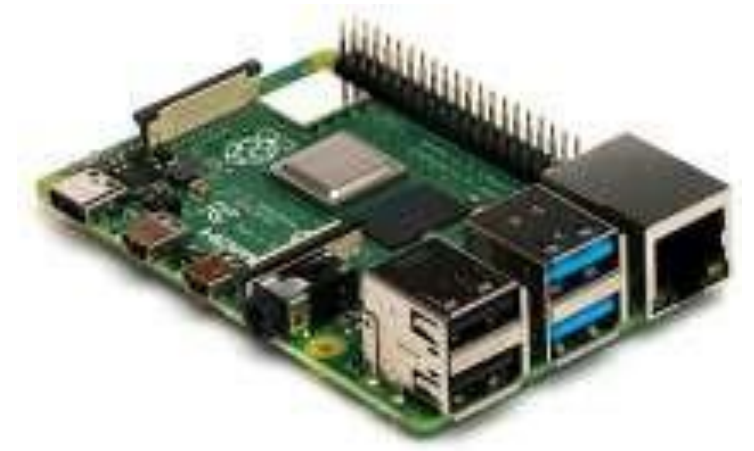

Figure 2: Raspberry Pi board

Raspberry Pi likely could be a progression of not many single-board PCs created at spans in the unified realm by the Raspberry Pi Foundation in relationship with Broadcom. The Raspberry $\mathrm{Pi}$ project initially inclined towards the advancement of showing fundamental designing in resources and non-industrial nations. The underlying model turned out to be further popular than expected, trade outside its objective commercial center for utilizes like AI. It's generally used in numerous spaces, as for climate recognition, inferable from its low worth, measured quality, and open vogue. It's normally used by PC and electronic specialists, because of its reception of HDMI and USB gadgets.

Published By:

Blue Eyes Intelligence Engineering and Sciences Publication (BEIESP)

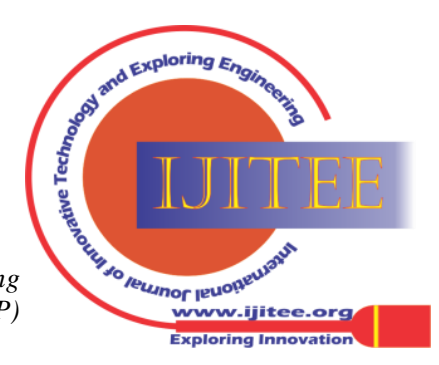




\section{Camera}

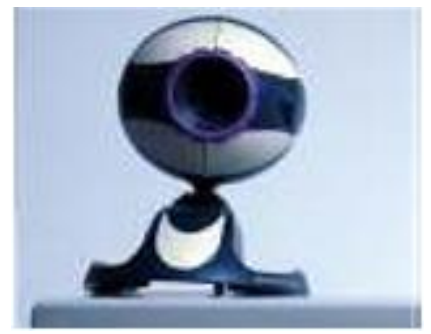

Figure 3: camera

A camera could likewise be a camcorder that feeds or transfers a picture or video in a period to or through an organization, similar to a web. Webcams space units region units commonly little cameras that sit on a table, append to a client's screen, or unit incorporated into the equipment. Webcams could likewise be utilized all through a video talk meeting including two or further of us, with discussions that embrace live sound and video.

\section{Ultrasonic Sensor}

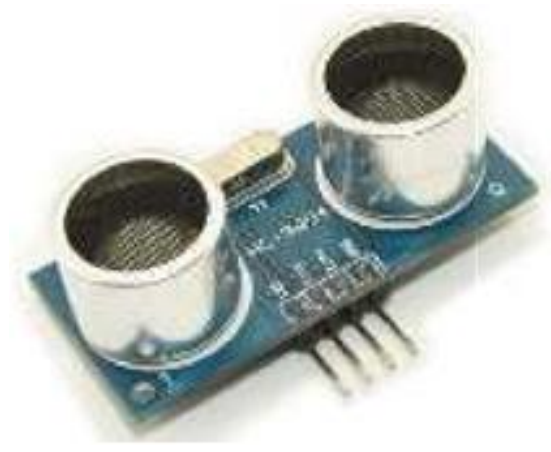

Figure 4: Ultra Sonic Sensor

Ultrasonic sensors notice the presence of people by causation out unintelligible sound waves into the region and estimation the speed at that they return.

\section{PIR Sensor}

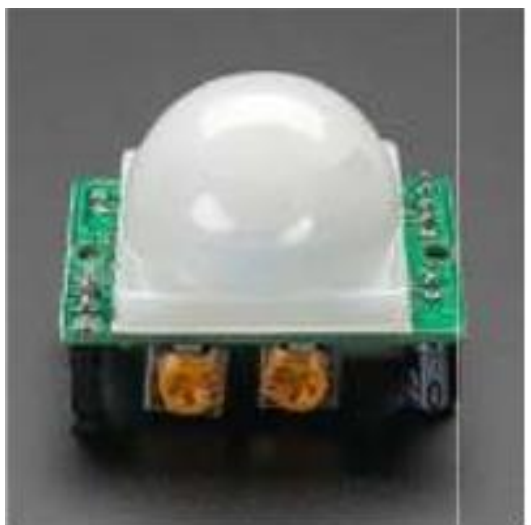

Figure 5: PIR Sensor

This is an adaptable Passive Infrared (PIR) gadget based around the BISS0001 PIR regulator. It grants you to line the affectability (up to 6 meters), beat time, and retrigger method of the movement gadget so you will be prepared to tailor-fit it to your application. Whenever movement is identified, the yield goes high $(3 \mathrm{~V})$.

\section{Speaker}

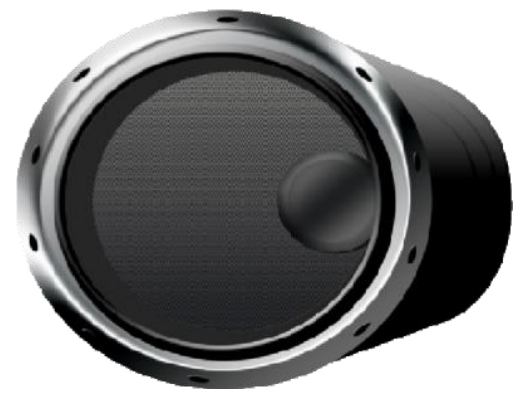

Figure 6: Speaker

Sound speakersare one of the free Car Speakers. This picture is straightforward foundation and Audio speakers free exchange,the sound speaker is one in all the concerning free auto Speaker. This picture might be a reasonable foundation and design.

\section{Software Requirements}

VNC likely could be a graphical work area sharing framework that permits you to distantly deal with the work area interface of one versatile PC (running VNC Server) from another compact PC or cell phone (running VNC Viewer). VNC Viewer sends the console and either mouse circles occasions to VNC Server and gets updates to the screen correspondingly. you'll see the work area of the Raspberry Pi at stretches a window on your versatile PC or cell phone. you might have the option to oversee it, for example, you were working on the Raspberry Pi itself.

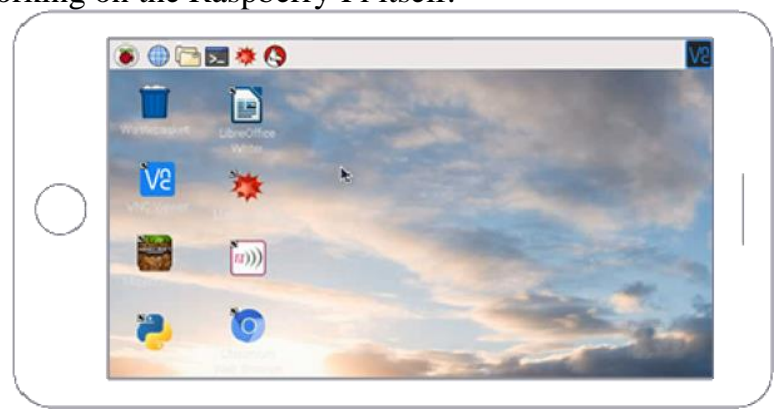

Figure 7: VNC Viewer

\section{EXPERIMENTAL RESULTS}

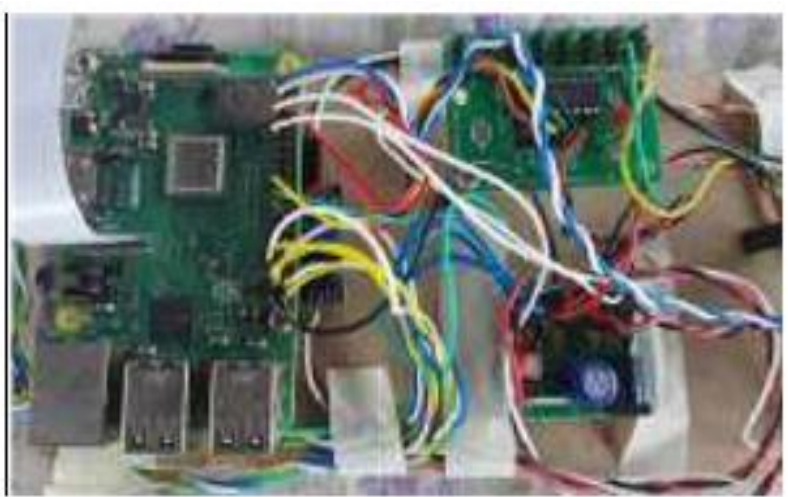

Figure 8: Hardware setup of proposed system

Published By:

Blue Eyes Intelligence Engineering and Sciences Publication (BEIESP)

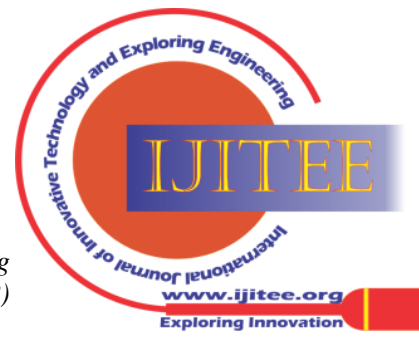




\section{A Smart Assistance System for Visually Impaired People}

The advanced camera catches a \{picture from a given picture and furthermore, the text from the caught picture is recovered into partner degree sound yield exploitation Raspberry Pi. Also, furthermore, we watch out for experience the space of the thing exploitation the indistinct detecting component. Each yields region unit is distinguished in partner degree sound.

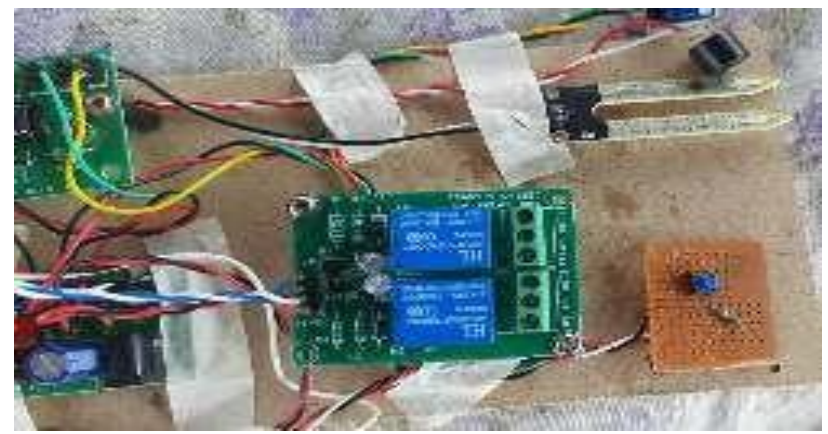

Figure 9: Hardware setup of Proposed System

Fig2 shows the parts that region unit mounted on the perf board. This gadget comprises of an electrical gadget and a rectifier that is used to venture down the AC voltage and convert AC into required DC. RF transmitter is mounted on the perf board though the RF collector is framed independently and mounted on the white board.

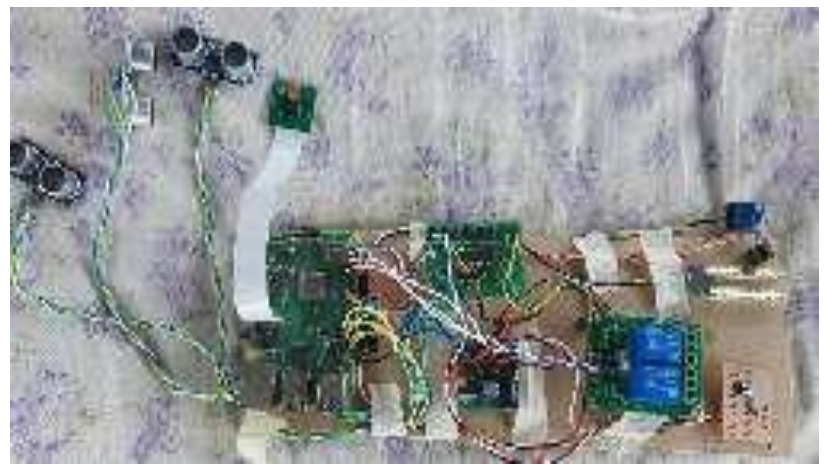

Figure 10: Hardware setup of Proposed System

Fig3 Shows, the unhearable detecting component is mounted with the stick through that impediments region unit distinguished. A water sensor is what's more mounted at the lower part of the accompanying sight the presence of burrows and water on a superficial level.

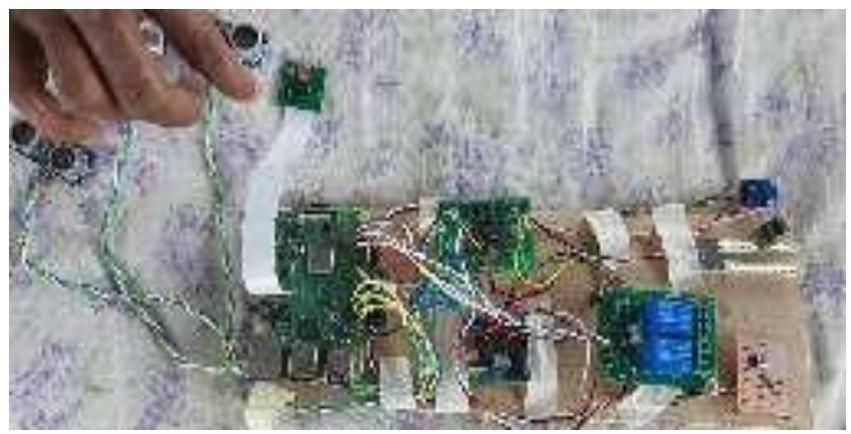

Figure 11: Hardware setup of Proposed System

An unhearable detecting component with the model, HC-SR04 is carried out all through this gadget as displayed in fig4. It's familiar with ensuring the place of the impediment. For impermanent distance obstructions, a partner degree partner infrared sensor is used that is mounted close to the

Published By:

Blue Eyes Intelligence Engineering and Sciences Publication (BEIESP) (C) Copyright: All rights reserved.

quiet sensor. This sensor could likewise be a particularly standard sensor used in numerous applications where measure distance or detecting objects square measure required.

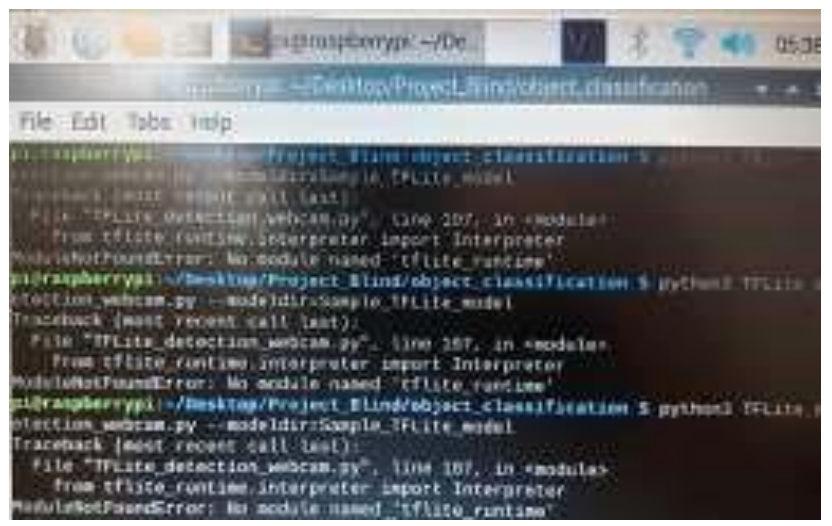

Figure 12: Software Results of Proposed System

The fig. five shows the sequential yield of the indistinct gadget in code. When the impediment is identified by the gadget, it shows the perusing inside the chronic screen with the house between the obstructions and in this way the gadget. Seeing the house the perusing differs.

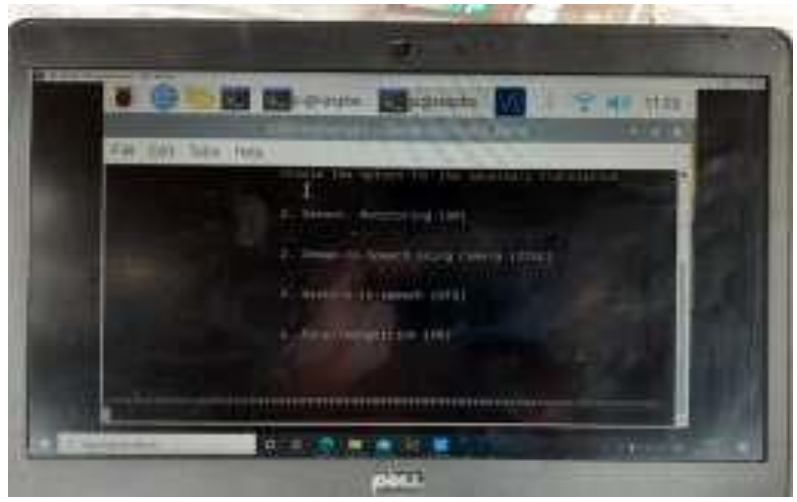

Figure 13: Software Results of Proposed System

The above fig. 6 shows the chronic screen perusing of the detecting of the water indicator. When the condition comes true with the indicator the yield "water identified" shows on the chronic screen. The code is written in a ton of the strategies that if the condition comes in-tuned with the water finder signal sound is produced from the ringer.

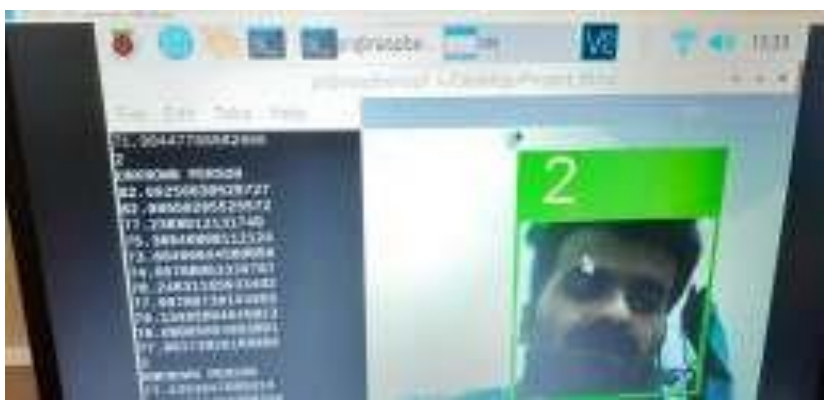

Figure 14: Software Results of Proposed System

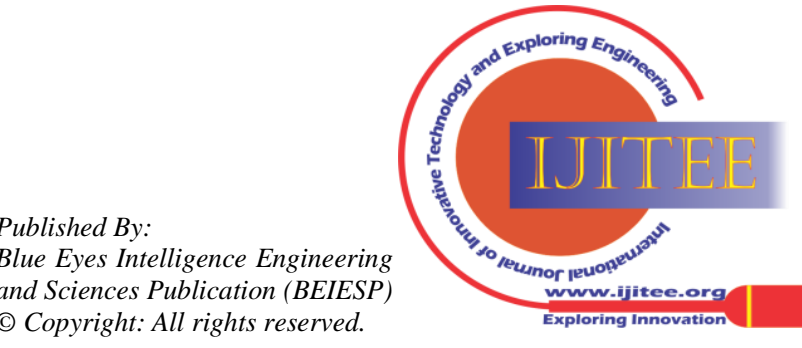


The fig.7 shows the chronic screen perusing of the detecting of the water detecting component.

\section{CONCLUSION}

We have authorized partner picture to-discourse transformation procedure exploitation raspberry pi. The reenactment results square measure effectively checked thus the equipment yield has been tried exploitation entire various examples. Our algorithmic program effectively measures the picture and peruses it out unmistakably. typically|this can be regularly proficient conjointly as partner degree partner conservative gadget for outwardly hindered individuals. We've applied our algorithmic program to numerous photographs and settled that it effectively can its transformation. The gadget is minimized and supportive to society.

\section{REFERENCES}

1. Ray Kurzweil KReader Mobile User Guide, knfb Reading Technology INC. (2008). [Online]. Available:

2. http://www.knfbReading.com

3. Ms.AthiraPanicker good looking assistant label reading system with voice output for the blind mistreatment raspberry pi, Ms.Anupama Pandey, Ms.VrunalPatil YTIET, University of metropolis ISSN: 2278 - 1323 International Journal of Advanced analysis in pc Engineering \& Technology (IJARCET) Vol. 5, Issue 10, October 20162553 WWW.ijarcet.org.

4. MarutTripathi, Manish Kumar, Vivek Kumar,Warshakandlikar A Navigation System for blind folks International journal for analysis in the field of study and engineering technology (erased) - Vol. 2 issues 4, Jul-Aug 2014.

5. DimitriosDakopoulos and Nikolaos G.Bourbakis wearable Obstacle dodging Electronic Travel Aids for Blind IEEE Transactions on systems, man and information science, half C (Applications and Reviews). Vol. 40, issue 1, Jan 2010.

6. Xilin Chen, Jie Yang, Jing Zhang, and Alex Waibel Automatic Detection and Recognition of Signs From Natural Scenesieee transactions on image process, Vol. 13, NO. 1, Jan 2004.

7. William A. Ainsworth A system for changing English text into speech IEEE Transactions on Audio and Electroacoustics, Vol. 21, Issue 3, Jun 1973.

8. Zoran Zivkovic. Improved accommodative Gaussian Mixture Model for Background Subtraction Pattern Recognition, 2004. ICPR 2004. Proceedings of the seventeenth International Conference on twenty Gregorian calendar month 2004.

9. S. B. Shorte, Mandar Deshpande, Prashant Deshmukh, SanjaykumarMathapati helpful Translator for Deaf \& Dumb folks IJECCE Vol. 5, Issue four Gregorian calendar months, Technovision-2014.

10. Michael McEnancy Finger Reader Is an Associate in nursing audio reading contraption for finger IJECCE Vol. 5, Issue four July-2014.

11. Vasanthi. G and Ramesh adult male Y Asian country. Vision-Based helpful System for Label Detection with Voice Output, Department of ECE, DMI faculty of Engineering, Chennai, Jan 2014.

\section{AUTHORS PROFILE}

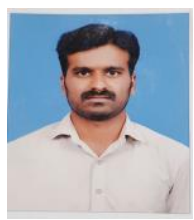

Mr. Muppla Jagadeesh, M.Tech (Embedded systems) from Kuppam Engineering College, Kuppam. he's conjointly Attended varied conferences, workshops, International Seminars, and school organized Industrial Visits. His areas of interest are Digital natural philosophy, IoT, and Embedded Systems.

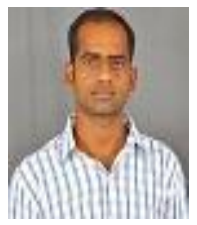

Mr. P Ajay Kumar Reddy, M.Tech.,(Ph.D.), is working as an associate prof within the Department of ECE, Kuppam Engineering College, Kuppam. He has organized Entrepreneurship Orientation Program and workshops and MATLAB Tools. His areas of interest are Digital natural philosophy and Communication Systems, IoT, and Embedded Systems.

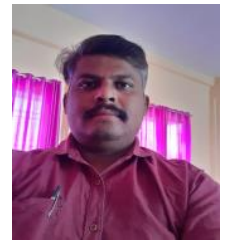

Dr. S. Nanda Kishor, M.Tech., Ph.D., is working as an associate prof within the Department of ECE, Kuppam Engineering College, Kuppam. He's a member of the Society of Digital data and Wireless Communication, the International Association of Engineers, and also the Institute of Engineers and Indian for Technical Education. He has organized Entrepreneurship Orientation Program and workshops and MATLAB Tools. His areas of interest are Digital natural philosophy and Communication Systems, IoT, and Embedded Systems.

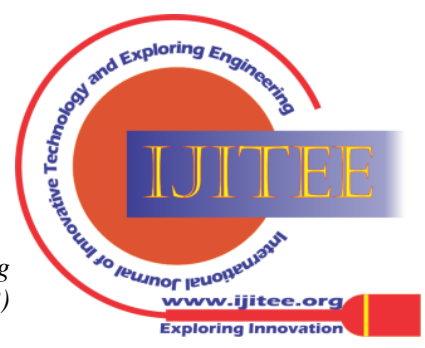

